\title{
Adaptive changes in physiological and perceptual responses during 10-day heat acclimation training using a water-perfused suit
}

Yelin $\mathrm{Ko}^{1}$, Seon-Hong Seol ${ }^{1}$, Juho Kang ${ }^{1,2}$ and Joo-Young Lee ${ }^{1,3^{*}}$

\begin{abstract}
Background: While active heat acclimation strategies have been robustly explored, not many studies highlighted passive heat acclimation strategies. Particularly, little evidence demonstrated advantages of utilizing a waterperfused suit as a passive heating strategy. This study aimed to explore heat adaptive changes in physiological and perceptual responses during 10-day heat acclimation training using a water-perfused suit.

Methods: Nineteen young males were divided into three experimental groups: exercise condition $\left(N=6, H A_{E X E}\right.$, 1 $\mathrm{h}$ exercise at $6 \mathrm{~km} \mathrm{~h}^{-1}$ followed by 1 -h rest in a sitting position), exercise and passive heating condition ( $N=6$, HA $A_{\text {EXE+SUIT, }}$ 1-h exercise at $6 \mathrm{~km} \mathrm{~h}^{-1}$ followed 1-h passive heating in a sitting position), and passive heating condition ( $N=7, H A_{\text {suIr, }}$ 2-h passive heating in a sitting position). All heating programs were conducted for 10 consecutive days in a climatic chamber maintained at $33{ }^{\circ} \mathrm{C}$ with $60 \%$ relative humidity. The passive heating was conducted using a newly developed water-perfused suit with $44^{\circ} \mathrm{C}$ water.

Results: Greater whole-body sweat rate and alleviated perceptual strain were found in HA $\mathrm{A}_{\text {SUIT }}$ and HA $\mathrm{A}_{\text {EXE+SUIT }}$ after 5 and/or 10 days $(P<0.05)$ but not in the exercise-only condition $\left(H A_{E X E}\right)$. Lower rectal temperature and heart rate were found in all conditions after the training $(P<0.05)$. Heat adaptive changes appeared earlier in $\mathrm{HA}_{\text {suIT }}$ except for sweat responses.

Conclusions: For heat acclimation in hot humid environments, passive and post-exercise heat acclimation training using the suit (water inflow temperature $44^{\circ} \mathrm{C}$ ) were more effective than the mild exercise $(1-\mathrm{h}$ walking at $6 \mathrm{~km}$ $\left.\mathrm{h}^{-1}\right)$. This form of passive heating $\left(\mathrm{HA}_{\text {suIT }}\right)$ may be an especially effective strategy for the elderly and the disabled who are not able to exercise in hot environments.
\end{abstract}

Keywords: Heat acclimation, A water-perfused suit, Passive heat acclimation, Post-exercise passive heating, Wholebody sweat rate, Rectal temperature, Heart rate

\footnotetext{
* Correspondence: leex3140@snu.ac.kr

'Department of Textiles, Merchandising and Fashion Design, College of Human Ecology, Seoul National University, COMFORT Laboratory, Bld. \# 222-Rm. \# 306, 1 Gwanak-ro, Gwanak-gu, Seoul 08826, Korea

${ }^{3}$ Research Institute of Human Ecology, Seoul National University, Seoul, Korea

Full list of author information is available at the end of the article
}

(c) The Author(s). 2020 Open Access This article is licensed under a Creative Commons Attribution 4.0 International License, which permits use, sharing, adaptation, distribution and reproduction in any medium or format, as long as you give appropriate credit to the original author(s) and the source, provide a link to the Creative Commons licence, and indicate if changes were made. The images or other third party material in this article are included in the article's Creative Commons licence, unless indicated otherwise in a credit line to the material. If material is not included in the article's Creative Commons licence and your intended use is not permitted by statutory regulation or exceeds the permitted use, you will need to obtain permission directly from the copyright holder. To view a copy of this licence, visit http://creativecommons.org/licenses/by/4.0/. The Creative Commons Public Domain Dedication waiver (http://creativecommons.org/publicdomain/zero/1.0/) applies to the data made available in this article, unless otherwise stated in a credit line to the data. 


\section{Introduction}

Water-perfused suits have been widely used in thermophysiological studies to form an artificial microclimate on the skin for inducing cold stress [1], heat stress [2], or both [3]. Since circulating water temperature can be easily controlled inside such a suit to directly cool and/ or heat the skin, this kind of clothing can be an effective whole-body hypothermia or hyperthermia induction method. Previous investigations on the thermophysiological effects of water-perfused suits included focuses on age-related differences in cardiac functions [4], thermosensitivity of peripheral skin sites [5], vascular responses [6], and metabolic responses [7]. Furthermore, passive heating using the suit has also been investigated to determine whether it is effective for treating cancer [8] or symptomatic peripheral artery disease [9].

Despite its extensive use in manipulating skin and body core temperatures $\left(T_{\text {core }}\right)$, to the best of our knowledge, there is little research highlighting the potential benefits of utilizing a water-perfused suit as a heat acclimation (HA) strategy. Typically, exercising in heat (active HA strategy) is the most commonly accepted method to induce the following HA responses [10], all of which contribute to improved heat tolerance: (1) decrease in body core temperature; (2) reduction in heart rate; (3) lessened physiological strain index (PSI); (4) increase in sweat rate; (5) alleviated perceptual strain. Other studies advocated passive HA strategies, subjecting individuals to heat stress without any exercise mainly by immersion in hot water [11], entering a heat chamber [12], or sauna [13]. These studies suggested that active strategies are not necessary to induce $\mathrm{HA}$ as the main stimulus for heat adaptation is simply a repeated rise in body core temperature [14]. However, passive heating has been preferred less because it is not considered as effective as exercise to induce heat acclimation; thus passive heating has often been recommended post-exercise $[14,15]$.

Donning a water-perfused suit has proved a powerful method to elevate body core temperature which is the requirement for $\mathrm{HA}$ and may have the following advantages. First, during passive heating, heat flow through the suit can be continuously monitored. A vaporimpermeable layer, which can be an outer layer of the suit, makes it into a non-evaporative microclimate. Therefore, when heat is lost from the skin, it is considered to result only from dry heat transfer [16]. From calculated heat flow during passive HA training, individual heat storage can be estimated which may make comparisons between active and passive strategies easier. Second, donning a water-perfused suit may be a superior strategy in hot humid environments. According to Périard et al. [17], effects of humidity on HA are highly likely to exist because of the physiological and biophysical differences between the two kinds of heat. According to these authors, to attain a high rate of evaporative cooling in a hot humid environment, it is imperative to keep a higher skin temperature or a larger wetted skin area than in a dry environment. Both conditions can be readily met inside the enclosure of a skin-heating waterperfused suit, which might reinforce the rationale for choosing this newly proposed method to improve heat tolerance to humid summer climates, like those of South Korea or Japan. Last but not the least, development of a novel HA method with no exercise can expand the application of HA to the disabled or the old who are not in a condition to exercise in heat.

This study explored heat adaptive changes in physiological and perceptual responses during 10-day heat acclimation training using a water-perfused suit. Changes elicited by passive and post-exercise HA strategies utilizing a water-perfused suit were compared to those obtained by a mild exercise based, active strategy. The present study hypothesized that (1) donning a water-perfused suit as passive and post-exercise strategies would both induce heat adaptive changes during the assigned intervention, and (2) differential effects of HA induction between the passive and post-exercise strategies would exist.

\section{Methods \\ Subjects}

Nineteen young Korean males participated in the present study. The subjects were all non-athletes. Anyone having cardiovascular, respiratory, or heat-related illnesses or symptoms were excluded in the recruiting process. During the 10-day HA program, each subject was instructed to refrain from strenuous exercise as well as alcohol intake and not to eat any food before arriving at the laboratory. The aims, procedures, discomforts, and risks of the present study were explained to subjects before the first day of the experiments, and they signed to an informed consent form. The current study was approved by the Institute Review Board of Seoul National University (IRB No. 1905/003008).

\section{Development of a water-perfused suit and the donning process}

A newly developed water-perfused suit was utilized in the experiments (Fig. 1). The suit consisted of a longsleeve jacket and calf-length pants. The pants were cropped to make the donning process quicker. For easy washing, the inner layer (nylon-spandex mesh: nylon $85 \%$ and polyurethane $15 \%$ ) and outer layer (polyester $92 \%$ and polyurethane $8 \%$ ) were designed to be detachable. A total of $30.9 \mathrm{~m}$ of PVC tubing (inner diameter 4 $\mathrm{mm}$ and outer diameter $6 \mathrm{~mm}$ ) was inserted into the inner layer in the chest, abdomen, upper and lower back, and front and back thigh. Peripheral body sites (hands, 


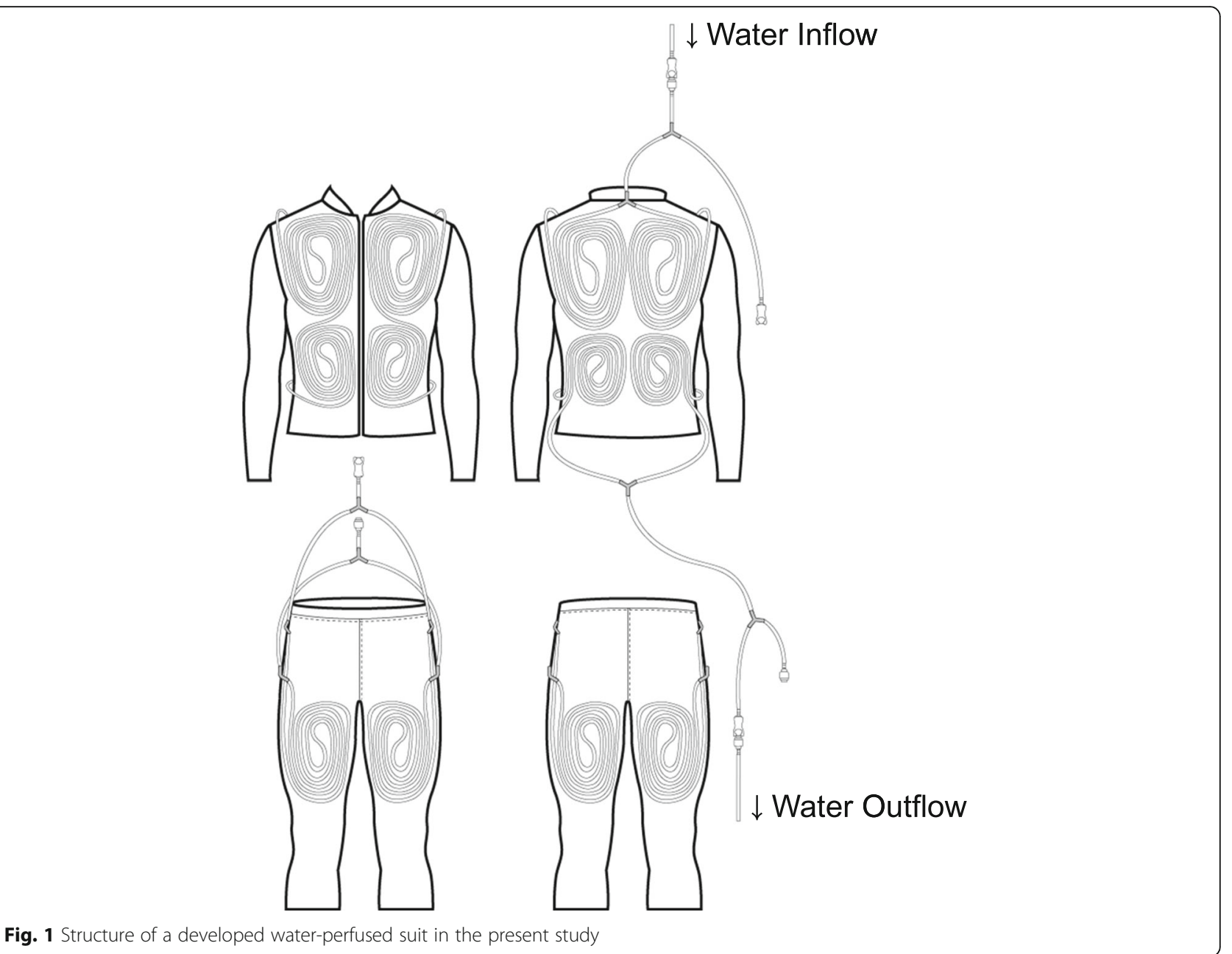

arms, legs, and feet) were not heated in order to optimize the water circulation rate. The suit was connected to a water bath circulator to heat the skin (RW0525G, JEIO TECH, Korea, a resolution of $0.1{ }^{\circ} \mathrm{C}$ ), with an average water flow rate of $37.31 \pm 0.86 \mathrm{~L} \mathrm{~h}^{-1}$.

The donning process included the following steps: Firstly, after wearing the jacket and the cropped pants, subjects wore knee-length sports socks to cover their calves. Secondly, to enhance the efficiency of heat transfer from the circulating water to the skin, the outer layer of the jacket was pinned so that the suit tightly fit to each subject's body. Two elastic bands (one around the chest and the other around the waist) were additionally worn to press the tubing against the skin. Thirdly, a vaporimpermeable insulating jacket and pants (polyester 100\%, PVC coating) were donned over the suit to minimize evaporative and radiant heat loss from the body.

\section{Experimental conditions: 10-day heat acclimation intervention}

The 10-day HA intervention consisted of three experimental conditions. Subjects were randomly assigned to one of the three experimental conditions and participated in the assigned 2-h program. Each subject was trained for their assigned experimental condition which they followed for their 10-day HA intervention. Subjects were evenly distributed in terms of age, height, body weight, body surface area, perceived heat tolerance, and maximal oxygen uptake $\left(V \mathrm{O}_{2 \max }\right)$ into three groups (Table 1). The first group, $\mathrm{HA}_{\mathrm{EXE}}(N=$ $6)$, was a mild exercise group. They completed a 1-h treadmill-walking exercise session at $6 \mathrm{~km} \mathrm{~h}^{-1}$ with $0 \%$ grade followed by a $1-\mathrm{h}$ seated rest. The second group, $\mathrm{HA}_{\mathrm{EXE}+\mathrm{SUIT}}(N=6)$, finished the same exercise session as $\mathrm{HA}_{\mathrm{EXE}}$ did but further, post-exercise, donned the suit for another hour while sitting on a chair. The third group, HA $\mathrm{H}_{\text {SUIT }}(N=7)$, wore a water-perfused suit for $2 \mathrm{~h}$ without any exercise. The exercise intensity $\left(6 \mathrm{~km} \mathrm{~h}^{-1}\right.$ with $0 \%$ grade) for $\mathrm{HA}_{\mathrm{EXE}}$ and $\mathrm{HA}_{\mathrm{EXE}+\mathrm{SUIT}}$ corresponded to $39 \pm 4 \% \mathrm{HR}_{\max }$, which was estimated by the age of the subjects. Water inflow temperature $\left(T_{\text {wi }}\right)$ was $44.2 \pm 0.1{ }^{\circ} \mathrm{C}$ for $\mathrm{HA}_{\text {EXE+SUIT }}$ and $44.3 \pm 0.1{ }^{\circ} \mathrm{C}$ for $\mathrm{HA}_{\text {SUIT }}(P=$ 0.425). 
Table 1 Characteristics of subjects in each experimental group

\begin{tabular}{lllll}
\hline & $H_{\text {EXE }}(N=6)$ & $H_{\text {EXE }+ \text { SUIT }}(N=6)$ & $H A_{\text {SUIT }}(N=7)$ & $P$ value \\
\hline Age $($ years $)$ & $22 \pm 2$ & $22 \pm 2$ & $24 \pm 2$ & $175.7 \pm 6.0$ \\
Height $(\mathrm{cm})$ & $174 \pm 2.2$ & $174.8 \pm 5.0$ & $71.7 \pm 10.4$ & N.S. \\
Body weight $(\mathrm{kg})$ & $69.5 \pm 7.9$ & $69.1 \pm 10.2$ & $1.9 \pm 0.1$ & N.S. \\
Body surface area $\left(\mathrm{m}^{2}\right)^{\mathrm{a}}$ & $1.9 \pm 0.1$ & $1.9 \pm 0.1$ & $4 \pm 1$ & N.S. \\
Perceived heat tolerance & $3 \pm 2$ & $3 \pm 2$ & $47.5 \pm 1.5$ & N.S. \\
$\mathrm{VO}_{2 \text { max }}\left(\mathrm{mL}\right.$ 'min $\left.\mathrm{kg}^{-1}\right)$ & $47.5 \pm 9.1$ & $46.6 \pm 6.8$ & N.S. \\
\hline
\end{tabular}

All data were expressed as mean \pm SD (standard deviation)

${ }^{a}$ Body surface area was estimated using the equation from Lee et al. [18]

${ }^{b}$ Perceived heat tolerance was attained with following 7-point scale: 1,1 am very susceptible to heat; 2,1 am susceptible to heat; 3 , I am a little susceptible to heat; 4, I am neither susceptible nor tolerant to heat; 5,1 am a little tolerant to heat; 6,1 am tolerant to heat; 7 , I am very tolerant to heat

\section{Experimental procedures}

Each subject visited the laboratory in ten consecutive mornings to participate in the daily HA training program. Arriving at the laboratory at the scheduled time, subjects first took a rest drinking $300 \mathrm{~mL}$ of water. Identical, size appropriate undershorts, shorts, and socks were provided as experimental clothing before instrumentation. Subjects were instructed to bring their own running shoes every day. Subjects in $\mathrm{HA}_{\mathrm{EXE}}$ and $\mathrm{HA}_{\mathrm{EXE}+\mathrm{SUIT}}$ waited in the preparation room wearing the set of experimental clothing before beginning their trial. By contrast, subjects in HA $\mathrm{A}_{\text {SUIT }}$ took off their shorts and changed into a water-perfused suit approximately 10 min before their trial. For each trial, baseline rectal temperatures and heart rates were checked to make sure the values were within normal ranges before entering the climatic chamber (maintained at $33{ }^{\circ} \mathrm{C}$ with $60 \%$ relative humidity).

After $10 \mathrm{~min}$ of sitting, the 2-h HA program started according to each subject's assigned experimental condition. For the first $1 \mathrm{~h}$ of the intervention, subjects in $\mathrm{HA}_{\mathrm{EXE}}$ and $\mathrm{HA}_{\mathrm{EXE}+\mathrm{SUIT}}$ walked at an intermittent rate (2 repeats of 25-min walking and 5-min break). During the first break, they took a seated rest. After finishing the second walking session, during the second 5-min break, $\mathrm{HA}_{\text {EXE+SUIT }}$ changed into a water-perfused suit and started resting as soon as the change was complete, while $\mathrm{HA}_{\mathrm{EXE}}$ maintained a standing posture before starting a seated rest for the remainder of the hour. No additional break to make up for the time it took for the change of clothes was not given to the subjects in $\mathrm{HA}_{\mathrm{EXE}+\mathrm{SUIT}}$ to equalize the duration exposed to the warm humid environment $(2 \mathrm{~h})$, between the groups. $\mathrm{HA}_{\mathrm{EXE}+\mathrm{SUIT}}$ 's post-exercise passive heating session lasted for the second $1 \mathrm{~h}$ of the program (2 repeats of 25-min heating and 5-min break) and was initiated by connecting the circulating water bath to the suit to allow inflow of warm water. Under the post-exercise passive heating condition, a break was given by disconnecting the suit from the circulating water and by instructing the subjects to unzip the insulating jacket. Subjects in HA $\mathrm{A}_{\text {SUIT }}$ experienced the passive heating protocol using a waterperfused suit for $2 \mathrm{~h}$ (4 repeats of 25-min heating and 5min break). During the break, they took the same type of the break as $\mathrm{HA}_{\mathrm{EXE}+\mathrm{SUIT}}$ in the post-exercise heating session. All subjects drank a total of $600 \mathrm{~mL}$ of water ad libitum during the $2 \mathrm{~h}$. It was not until the 2-h HA program was terminated that subjects were asked to leave the climatic chamber.

\section{Measurements and calculations}

Rectal temperature $\left(T_{\text {re }}\right)$ was measured using a rectal probe inserted $16 \mathrm{~cm}$ beyond the anal sphincter and recorded every $5 \mathrm{~s}$ by a data logger (LT-8A, Gram Corporation, Japan). Heart rate was monitored every $1 \mathrm{~s}$ using a polar electrode with a chest belt (RC3 GPS, Polar Electro, Finland), and the data were sorted out at the interval of $5 \mathrm{~s}$. Whole-body sweat rate was estimated using a change in total body mass which was measured in a semi-nude state on a calibrated scale before and after the experiment (ID2, Mettler-Toledo, Germany: resolution of $1 \mathrm{~g}$ ). As all subjects were required to drink 600 $\mathrm{mL}$ of water in each trial, $600 \mathrm{~g}$ was subtracted from each subject's post-experiment body mass. The body mass loss due to respiratory water loss was not considered significant. Perceptual responses were obtained every 10 min using the following scales: 9-point thermal sensation (4: very hot, 3: hot, 2: warm, 1: slightly warm, 0 : neutral, -1 : slightly cool, -2 : cool, -3 : cold, and -4 : very cold) and 4-point thirst sensation (3: very thirsty, 2: thirsty, 1: a little thirsty, 0: not thirsty). Rating of perceived exertion (RPE) was obtained according to the Borg [19] scale, only for the $\mathrm{HA}_{\mathrm{EXE}}$ and HAEX+SUIT because no exercise was required for $\mathrm{HA}_{\mathrm{SUIT}}$. Physiological strain index (PSI) was calculated using the equation from Moran et al. [20] (Eq. 1). Peak PSI was calculated averaging the previous 5 - min data at the time the maximum appeared. Heat flow (HF) through the waterperfused suit during the passive heating session was calculated using the following heat flow equation (Eq. 2). Heat flow was then converted to watts $\left(1 \mathrm{kcal} \mathrm{h}^{-1}=\right.$ $1.163 \mathrm{~W})$. 
Physiological strain index (PSI)

$$
\begin{aligned}
= & 5\left(T_{\text {ret }}-T_{\text {re } 0}\right) \cdot\left(39.5-T_{\text {re } 0}\right)^{-1} \\
& +5\left(\mathrm{HR}_{\mathrm{t}}-\mathrm{HR}_{0}\right) \cdot\left(180-\mathrm{HR}_{0}\right)^{-1}
\end{aligned}
$$

where $T_{\text {ret }}$ is rectal temperature at time $t\left({ }^{\circ} \mathrm{C}\right), T_{\text {reo }}$ is rectal temperature at $0 \mathrm{~min}\left({ }^{\circ} \mathrm{C}\right), \mathrm{HR}_{\mathrm{t}}$ is heart rate at time $t(\mathrm{bpm})$, and $\mathrm{HR}_{0}$ is heart rate at $0 \mathrm{~min}(\mathrm{bpm})$.

$$
\begin{aligned}
& \text { Heat flow (HF) }\left(\mathrm{kcal} \mathrm{h}^{-1}\right) \\
& \quad=h_{\mathrm{w}} \cdot C_{\mathrm{w}} \cdot\left(T_{\mathrm{wi}}-T_{\mathrm{wo}}\right)
\end{aligned}
$$

where $h_{\mathrm{w}}$ is water flow rate of the suit (liters per hour), $C_{\mathrm{w}}$ is water specific heat $=1 \mathrm{kcal} \mathrm{kg}^{-1}{ }^{\circ} \mathrm{C}^{-1}, T_{\mathrm{wi}}$ is water inflow temperature $\left({ }^{\circ} \mathrm{C}\right)$, and $T_{\text {wo }}$ is water outflow temperature $\left({ }^{\circ} \mathrm{C}\right)$.

\section{Data analyses}

All data were expressed as mean and standard deviation (mean $\pm \mathrm{SD})$. All statistical analyses were undertaken using SPSS statistics 25.0 with the significance level at $P$ $<0.05$. After testing for normality and sphericity, data from HA training on days 1,5 , and 10 were analyzed by using either a one-way repeated measures ANOVA or Friedman test for comparisons across the training days. As a post hoc test, pairwise comparisons with False Discovery Rate (FDR) correction were used when needed. When presenting results of rectal temperature increases during the three heat acclimation protocols, one-way ANOVA with the Tukey test as a post hoc was conducted to compare the values between the groups. The upper subscript $\mathrm{a}, \mathrm{b}$, and $\mathrm{c}$ in the sentences of the result section represented significantly identical experimental conditions determined by the Tukey test for post hoc. For graphical and analytical purposes, continuously measured rectal temperature and heart rate data during the training were averaged into 5-min blocks.

\section{Results}

Heat flow through the water-perfused suit and increment in rectal temperature during the intervention

During the first $1 \mathrm{~h}$ of the passive heating, heat flow through the water-perfused suit in $\mathrm{HA}_{\text {SUIT }}$ was $102 \pm 8$ $\mathrm{W}$ on average (Table 2). The 10-day average heat flow calculated during the second $1 \mathrm{~h}$ was $101 \pm 9 \mathrm{~W}$ and 102 $\pm 7 \mathrm{~W}$ for $\mathrm{HA}_{\mathrm{EXE}+\mathrm{SUIT}}$ and HA $\mathrm{H}_{\text {SUIT }}$, respectively (Table 2 ). One hour of mild exercise elevated $T_{\text {re }}$ by $1.2 \pm 0.2$ ${ }^{\circ} \mathrm{C}^{\mathrm{a}}$ for $\mathrm{HA}_{\mathrm{EXE}}$ and $1.1 \pm 0.2{ }^{\circ} \mathrm{C}^{\mathrm{a}}$ for $\mathrm{HA}_{\mathrm{EXE}+\mathrm{SUIT}}$ respectively, while it was increased by $0.5 \pm 0.2{ }^{\circ} \mathrm{C}^{\mathrm{b}}$ by the passive heating in HA $\mathrm{HAIT}_{\text {SUT }}(P<0.001)$. During the second 1 h, $T_{\text {re }}$ decreased by $0.5 \pm 0.2{ }^{\circ} \mathrm{C}^{\mathrm{C}}$ in $\mathrm{HA}_{\mathrm{EXE}}$ while taking a seated-rest, it was further elevated by $0.5 \pm 0.2{ }^{\circ} \mathrm{C}^{\mathrm{b}}$ by post-exercise suit donning in $\mathrm{HA}_{\mathrm{EXE}+\mathrm{SUIT}}$, and in $\mathrm{HA}_{\text {SUIT }}$, it achieved $1.0 \pm 0.1{ }^{\circ} \mathrm{C}^{\mathrm{a}}(P<0.001)$. As a result,

\begin{tabular}{|c|c|c|c|c|c|}
\hline & Day 1 & Day 5 & Day 10 & 10-day average & $P$ value \\
\hline \multicolumn{6}{|c|}{ Heat insertion by circulating water inside the water-perfused suit } \\
\hline \multicolumn{6}{|c|}{ During the 1 st $1 \mathrm{~h}$ of the suit donning $(\mathrm{W})$} \\
\hline HA & $101 \pm 12$ & $108 \pm 9$ & $100 \pm 16$ & $102 \pm 8$ & N.S. \\
\hline \multicolumn{6}{|c|}{ During the $2 \mathrm{nd} 1 \mathrm{~h}$ of the suit donning $(\mathrm{W})$} \\
\hline HA EXE+SUIT & $97 \pm 16$ & $106 \pm 6$ & $100 \pm 16$ & $101 \pm 9$ & N.S. \\
\hline HA $A_{\text {SUIT }}$ & $94 \pm 16$ & $107 \pm 9$ & $104 \pm 12$ & $102 \pm 7$ & N.S. \\
\hline$P$ value & N.S. & N.S. & N.S. & N.S. & \\
\hline
\end{tabular}
net increase in rectal temperature was $0.7 \pm 0.1{ }^{\circ} \mathrm{C}^{\mathrm{b}}, 1.6$
Table 2 Heat flow through the water-perfused suit on days 1, 5, and 10

All data were expressed as mean \pm SD (standard deviation)

$\pm 0.2{ }^{\circ} \mathrm{C}^{\mathrm{a}}$, and $1.5 \pm 0.2{ }^{\circ} \mathrm{C}^{\mathrm{a}}$ for $\mathrm{HA}_{\mathrm{EXE}}, \mathrm{HA}_{\mathrm{EXE}+\mathrm{SUIT}}$, and $\mathrm{HA}_{\text {SUIT }}$ respectively $(P<0.001)$. Average of area under the curve (AUC) of $T_{\text {re }}$ for $\mathrm{HA}_{\text {EXE+SUIT }}(165.4 \pm 15.7)$ was significantly greater than AUC of HA $\mathrm{HAIT}_{\mathrm{SUT}}(142.5 \pm$ 20.6) $(P=0.048)$.

\section{Whole-body sweat rate}

Whereas $\mathrm{HA}_{\mathrm{EXE}}$ did not show any differences in wholebody sweat rate across the $\mathrm{HA}$ training days, $\mathrm{HA}_{\mathrm{EXE}+-}$ sUIT and HA SUIT $_{\text {displayed higher whole-body sweat rate }}$ on day 5 and/or day $10(P=0.013, P=0.003$, respectively) (Fig. 2). Compared to the first day of training, whole-body sweat rate in $\mathrm{HA}_{\mathrm{EXE}+\mathrm{SUIT}}$ on day 5 increased by $71.1 \pm 51.5 \mathrm{~g} \mathrm{~h}^{-1} \mathrm{~m}^{-2}(P=0.006)$ and on day 10 by $79.4 \pm 44.5 \mathrm{~g} \mathrm{~h}^{-1} \mathrm{~m}^{-2}(P=0.011)$ (Fig. 2). For HA SUIT, whole-body sweat rate increased on day 10: compared to day 1 it increased by $74.0 \pm 51.7 \mathrm{~g} \mathrm{~h}^{-1} \mathrm{~m}^{-2}(P=0.014)$ and to day 5 by $69.2 \pm 45.3 \mathrm{~g} \mathrm{~h}^{-1} \mathrm{~m}^{-2}(P=0.021)$.

\section{Perceptual responses}

The $\mathrm{HA}_{\mathrm{EXE}}$ training adversely affected thermal sensation at the end of the seated rest, displaying lowest thermal sensation on the first day $(P<0.05)$ (Fig. 3a). In $\mathrm{HA}_{\text {EXE+- }}$ sUIT and HA $\mathrm{A}_{\mathrm{SUIT}}$, on the contrary, subjects perceived themselves as being less hot after 10 days of HA training $(P<0.05)$ (Fig. 3a). Pairwise comparison with FDR

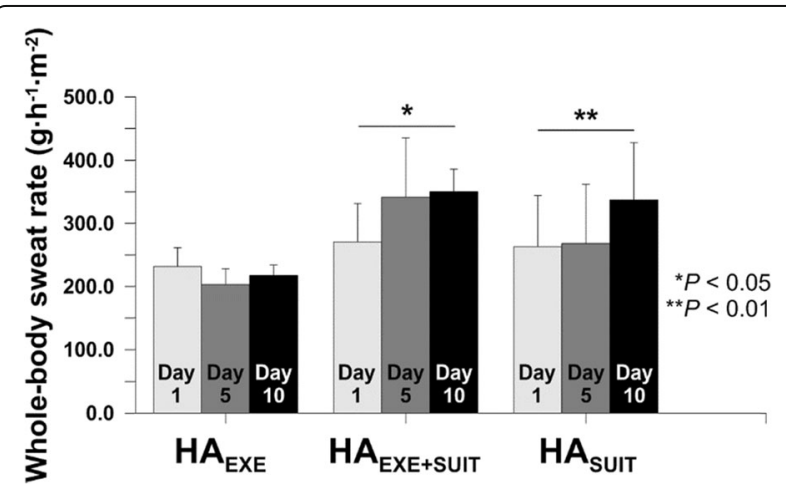

Fig. 2 Whole-body sweat rate on days 1, 5, and 10 for $\operatorname{HA}_{\text {EXE }}(N=6)$, $\operatorname{HA}_{\text {EXE }+ \text { SUIT }}(N=6)$, and HA $A_{\text {SUIT }}(N=7)$ 


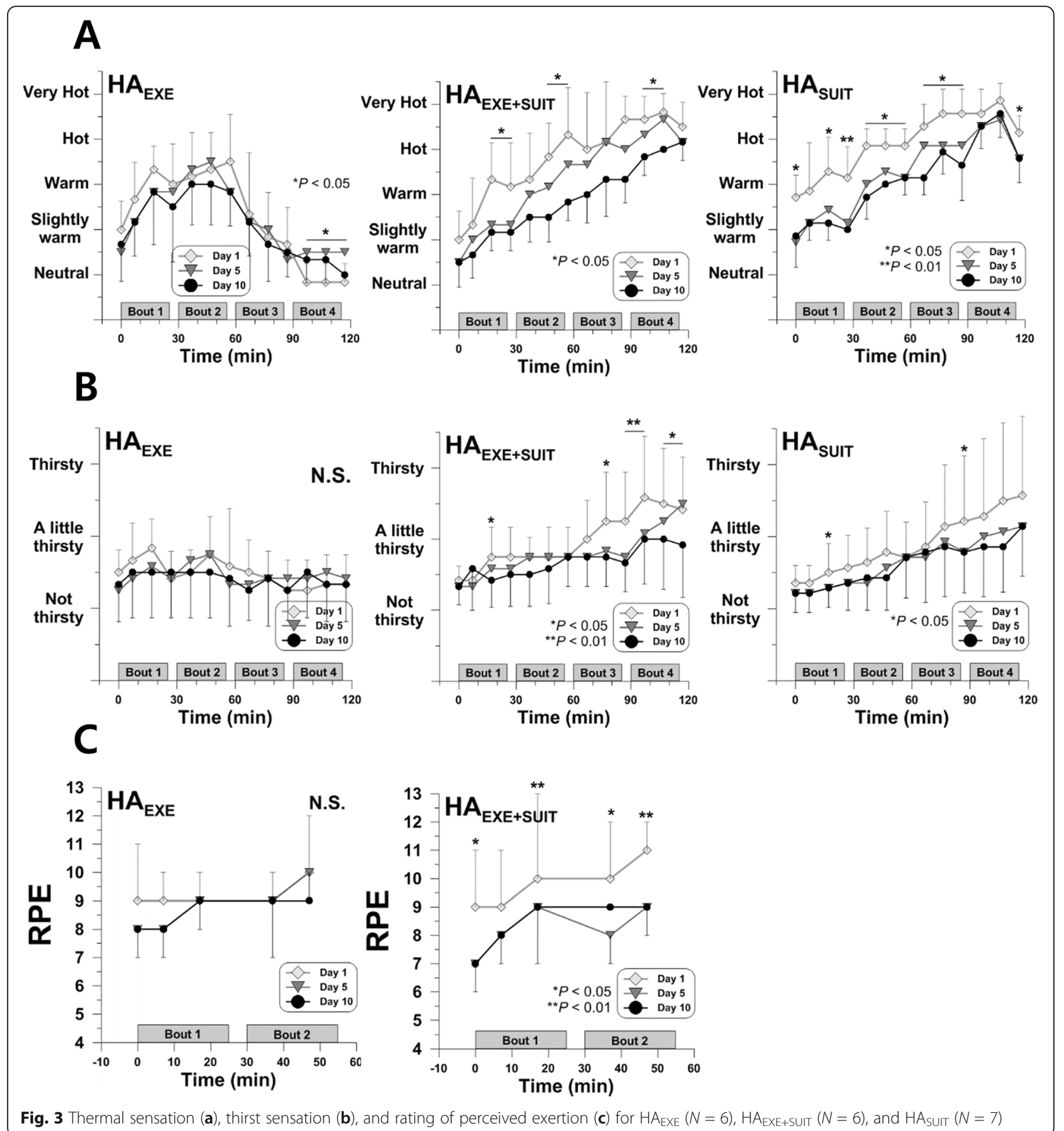

correction showed that thermal sensation in $\mathrm{HA}_{\text {SUIT }}$ on day 10 was significantly lower than the first day (27th min, $P=0.035$; 47th min, $P=0.042$ ). Thirst sensation did not change in $\mathrm{HA}_{\mathrm{EXE}}$ but decreased on day 5 and/or day 10 compared to day 1 in $\mathrm{HA}_{\mathrm{EXE}+\text { SUIT }}$ and HA $\mathrm{HAIT}_{\text {SUT }}(P$ $<0.05$ ) (Fig. 3b). Rating of perceived exertion (RPE) showed no differences after 10 days of HA training in $\mathrm{HA}_{\text {EXE}}$. Subjects in $\mathrm{HA}_{\mathrm{EXE}+\mathrm{SUIT}}$, however, reported lower RPE on day 5 and/or day 10 during the exercise session
$(P<0.05)$ (Fig. 3c). Pairwise comparisons with FDR correction showed that both on day 5 and day $10 \mathrm{RPE}$ at 47th min for $\mathrm{HA}_{\mathrm{EXE}+\mathrm{SUIT}}$ significantly dropped from day $1(P=0.021$ and $P=0.030$, respectively).

\section{Rectal temperature}

Lowest $T_{\text {re }}$ appeared on day 5 or day 10 in the different stages of the training protocols for $\mathrm{HA}_{\mathrm{EXE}}, \mathrm{HA}_{\mathrm{EXE}+\mathrm{SUIT}}$, and $\mathrm{HA}_{\text {SUIT. }}$. The active strategy-based mild exercise 
group $\mathrm{HA}_{\mathrm{EXE}}$ displayed lower rectal temperature on day 10 during the mid to last stage of exercise $(P<0.05)$ (Fig. 4a). Pairwise comparison with FDR correction revealed that in $\mathrm{HA}_{\mathrm{EXE}}, T_{\text {re }}$ of day 10 was lower than the values of day 1 (at 35th min, $P=0.038$ ) and lower than those of day 5 (25th to 35th min, $P<0.05$ ), respectively. For $\mathrm{HA}_{\mathrm{EXE}+\mathrm{SUIT}}$, significant differences across the days in $T_{\mathrm{re}}$ were present from the end of the exercise to the mid-stage of post-exercise suit donning $(P<0.05)$ (Fig. $4 \mathrm{~b})$. The HA $\mathrm{H}_{\text {SUIT }}$ showed the lowest $T_{\text {re }}$ at the end of the training $(P<0.05)$ (Fig. $4 \mathrm{c})$.

\section{Heart rate}

Heart rate on day 10 for $\mathrm{HA}_{\mathrm{EXE}}$ was significantly lower than day 1 and day 5 primarily during the seated rest period $(P<0.05)$ (Fig. 5a). According to the pairwise comparisons, heart rate for $\mathrm{HA}_{\mathrm{EXE}}$ on day 10 was lower than on day 1 (at 50th, 65th, 80th, 100th, and 120th min, $P<0.05$ ); it was also lower than heart rate on day 5 (at 100th min, $P=0.044$ ). When passive skin-heating was added post-exercise $\left(\mathrm{HA}_{\mathrm{EXE}+\mathrm{SUIT}}\right)$, heart rate on day 10 displayed lower values from the mid-stage of the exercise session to the earlier stage of passive HA session $(P<0.05)$ (Fig. 5b). The post hoc test showed that heart rate for $\mathrm{HA}_{\mathrm{EXE}+\mathrm{SUIT}}$ on day 10 significantly decreased from day 1 (20th to 55th and 70th min; $P<0.05$ ). There were also significant differences in heart rate for $\mathrm{HA}_{\mathrm{EXE}+\mathrm{SUIT}}$ between day 1 and day 5 (40th and 45th min, $P<0.05)$. Unlike the other two HA strategies, HA $\mathrm{A}_{\text {SUIT }}$ induced lower heart rate on day 5 at the later stage of skin-heating $(P<0.05)$ (Fig. 5c). Pairwise comparison with FDR correction indicated that on day 5 heart rate for $\mathrm{HA}_{\text {SUIT }}$ significantly dropped from day 1 (90th and 120th min, $P<0.05$ ) and from day 10 (85th, 115th, and 120th min, $P<$ $0.05)$.

\section{Physiological strain index (PSI)}

Significant differences between the heat acclimation training days in peak and mean PSI were only found for HA $_{\text {SUIT }}$ (Fig. 6a, b). Peak PSI for HA HUIT $_{\text {Su }}$ on day 5 was the lowest $(P<0.001)$, showing significant differences from the values on day $1(P=0.001)$ and on day $10(P=$ 0.018) (Fig. 6a). Compared to the other two days, mean PSI also displayed the lowest value on day $5(P=0.027)$ (Fig. 6b).

\section{Discussion}

Human body can suffer from physiological and perceptual burdens in a hot environment in which the body temperature has to be effectively regulated, to prevent heat-related illness and impaired endurance performance $[10,14]$. Being considered one of the most important intervention to reduce physiological strain in heat, various effective HA protocols have been suggested mostly based on exercising strategies. The present study aimed to propose the novel method to induce heat acclimation without exercise, which was wearing a skin-heating water-perfused suit to provide exogenous thermal loads to the body. The effectiveness of wearing the suit was proved by exploring physiological and perceptual changes during the 10-day HA trainings. There are two main findings of the current study. Firstly, both wearing the water-perfused suit after the $1-\mathrm{h}$ exercise and throughout the $2-\mathrm{h}$ protocol had more advantageous effects on inducing HA than the mild exercise HA strategy. Secondly, the HA responses that appeared in the passive and post-exercise HA groups differed in terms of the timing of the HA protocols and the training days.
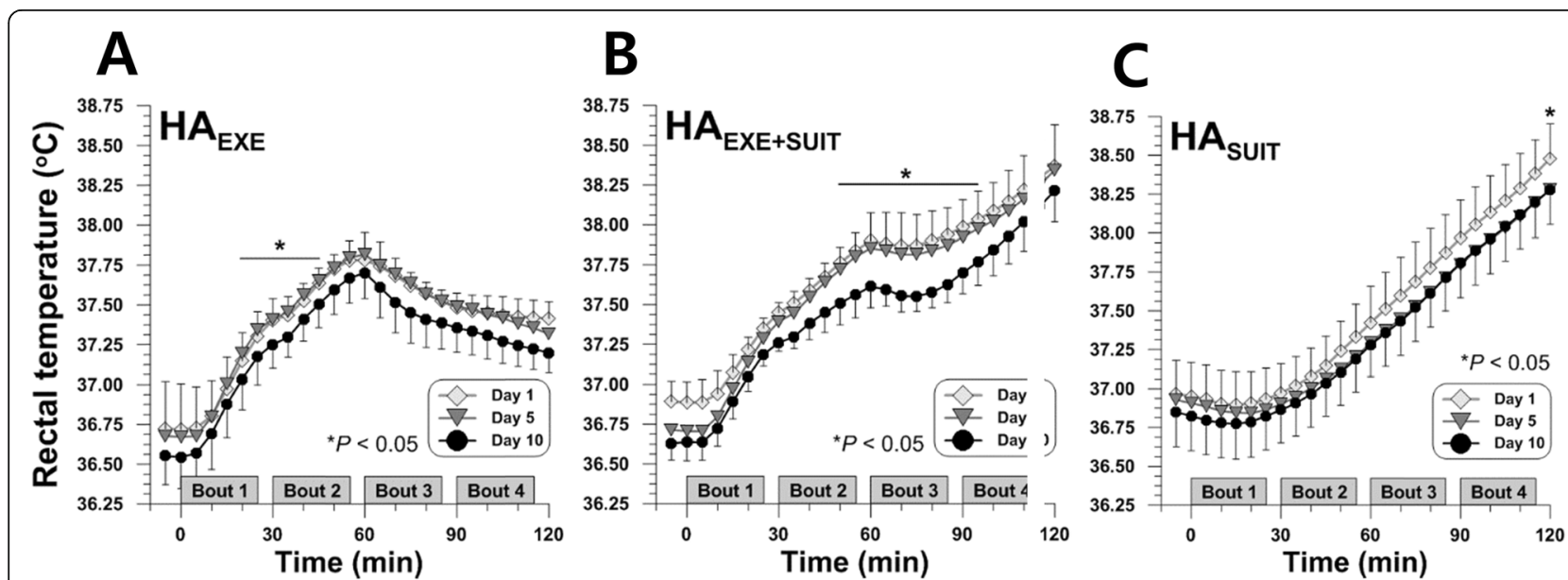

Fig. 4 Time courses of rectal temperatures during heat acclimation training on days 1, 5, and 10 for $H A_{E X E}(N=6)(\mathbf{a}), H A_{E X E+S U I T}(N=6)(\mathbf{b})$, and $\mathrm{HA}_{\text {SUIT }}(\mathrm{N}=7)(\mathbf{c})$ 

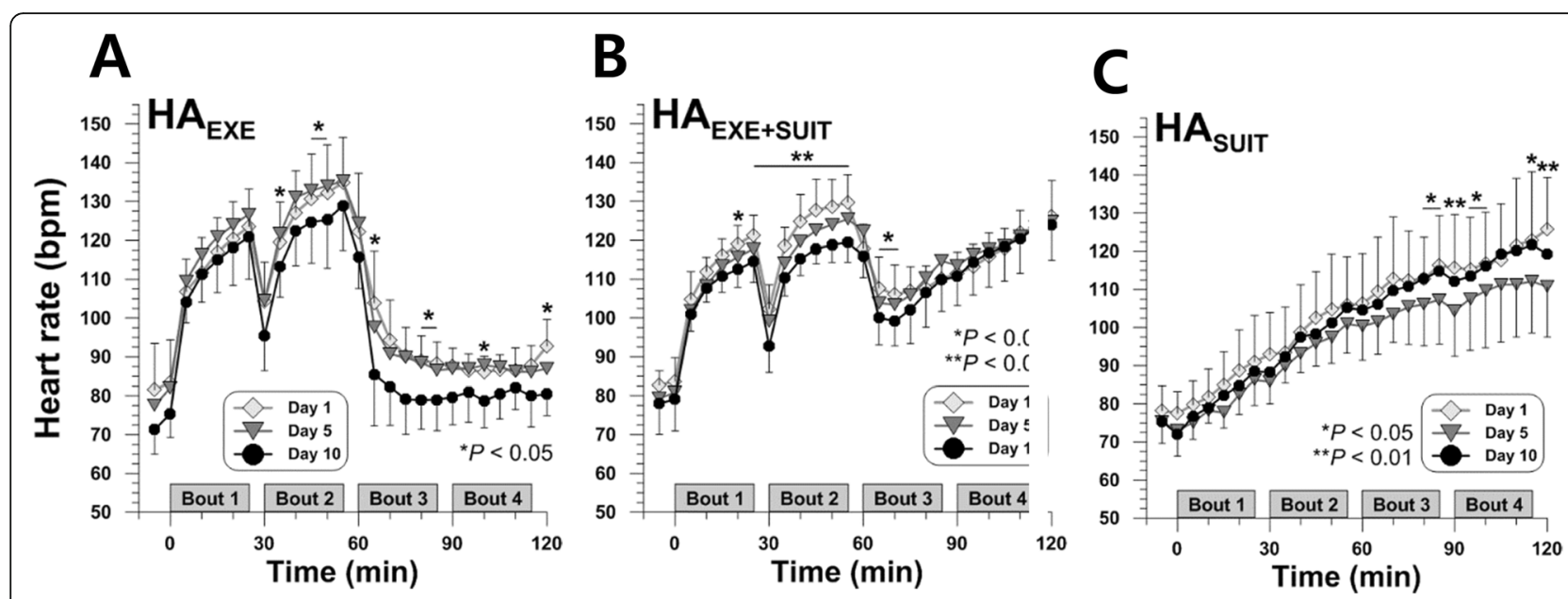

Fig. 5 Time courses of heart rate during heat acclimation training on days 1, 5, and 10 for $\operatorname{HA} A_{\text {EXE }}(N=6)(\mathbf{a}), H A_{\text {EXE+SUIT }}(N=6)(\mathbf{b})$, and HAsUIT $(N$ $=7)(\mathbf{c})$

Beneficial effects of passive and post-exercise heat acclimation training using a water-perfused suit When interpreting the present results, no significant differences in resting rectal temperature after training should be considered. Though the two HA strategies using a water-perfused suit induced "relatively" better responses than the mild exercise did, the extent of the obtained responses could be less than those reporting such differences. Whereas the mild exercise group $\left(\mathrm{HA}_{\mathrm{EXE}}\right)$ did not display any adaptive changes in whole-body sweat rate or perceptual responses, the other two HA strategies utilizing a water-perfused suit ( $\mathrm{HA}_{\mathrm{EXE}+\mathrm{SUIT}}$ and $\mathrm{HA}_{\text {SUIT }}$ ) induced $\mathrm{HA}$ through those adaptive changes after 5 days and/or 10 days of training. Considering each subject drank exactly the same amount of water during the training, lessened thirst sensation despite enhanced sudomotor capacity obtained in $\mathrm{HA}_{\mathrm{EXE+}}$ suIT and $\mathrm{HA}_{\text {SUIT }}$ is particularly noteworthy. Sweating during exercise in heat was known to increase plasma osmolality accompanied by plasma volume loss, stimulating thirst sensation for unacclimated individuals [21]. Rise in plasma volume induced by heat acclimation, which was large enough to keep plasma osmolality low despite increased sweating may explain attenuated thirst sensation with higher sweat rates. Though plasma volume was not directly measured in the present study, it could be expected to have happened based on the high correlation of 0.93 between increased plasma volume and elevations in sweat rate [22].

The advantages of donning the skin-heating suit after exercise on HA responses are in accordance with previous studies: it was suggested that post-exercise hot water immersion [23] or sauna bathing [13, 24] were more effective HA strategies than exercise alone. It is also worthy to note that this strategy was more effective in alleviating perceptual thermal strain not only during the
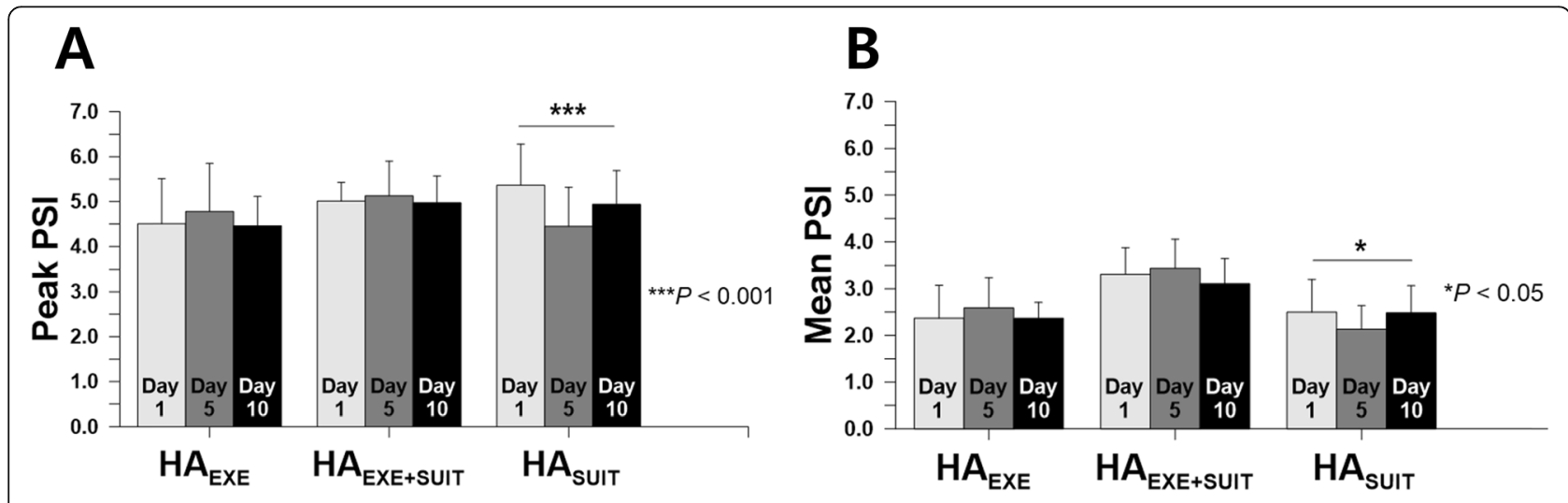

Fig. 6 Peak Perceptual Strain Index (a) and mean Perceptual Strain Index (b) on days 1, 5, and 10 for HAAXE $(N=6), H A_{E X E+S U I T}(N=6)$, and HA $(N=7)$ 
passive heating but also during exercise. Perceptual adaptations during exercise under heat stress are particularly important because those changes can relieve premature fatigue and exert positive influences on endurance performance [25-27]. In addition, heart rate for $\mathrm{HA}_{\text {EXE+SUIT }}$ was reduced after $\mathrm{HA}$ training primarily during exercise, while heart rate for $\mathrm{HA}_{\mathrm{EXE}}$ decreased mainly during the recovery. Lower heart rates coupled with lower $T_{\text {re }}$ found in $\mathrm{HA}_{\mathrm{EXE}+\mathrm{SUIT}}$ during exercise may be related with increased cardiac output, which can be possibly result from enhancement in plasma and stroke volume [28].

Passive strategies alone have been considered less effective for inducing HA than exercise-based strategies [10]. Even a review that highlighted the importance and practical values of passive HA protocols recommended these protocols be combined with exercise because exercise can lead to greater increases in body core temperature [14]. Daanen et al. [15] also pointed out that the positive adaptations induced by passive heat exposures, which are mainly related to blood volume, should be further compared and contrasted with actively induced HA responses. In contrast to the existing views, the findings of the present study suggest not only that donning a skinheating suit for $2 \mathrm{~h}$ can be a sufficiently effective HA strategy but also that such a strategy may be more effective than mild exercise in heat for $1 \mathrm{~h}$, at least in terms of activating sweat glands and alleviating perceptual strain as well as PSI. The effectiveness of wearing a water-perfused suit in inducing heat acclimation was, however, found to be better than only the mild exercise protocol walking at $6 \mathrm{~km} \mathrm{~h}^{-1}$ for $1 \mathrm{~h}$. Further investigation is needed on how its effects will be compared to higher intensity exercise conducted for longer duration.

In order to match the heat stress of passive and active HA strategies, the heat flow through the water-perfused suit should be calculated. The approximate $100 \mathrm{~W}$ of heat flow during the $1 \mathrm{~h}$ of suit donning corresponds to the work rate that has been known to raise mean body temperature by $1.3{ }^{\circ} \mathrm{C}$ [29]. Given that $T_{\text {re }}$ increased by $1.2 \pm 0.2{ }^{\circ} \mathrm{C}$ during the first hour in $\mathrm{HA}_{\mathrm{EXE}}$ while walking at $6 \mathrm{~km} \mathrm{~h}^{-1}$ with no incline, the difference between the heat storage rates in the passive versus active HA strategies of the current study does not seem too great. Still, whether the heat storage rates for each strategy should be matched is questionable considering passive strategies directly heat the skin first whose temperature rise would affect the temperature of body core whereas exercise-based strategies produce endogenous heat.

\section{Differential effects of passive and post-exercise heat acclimation training using a water-perfused suit}

The two strategies heating the skin either after exercise or throughout the heat acclimation protocol induced
HA responses at different times and on different acclimation days. In the exercise followed by passive HA, $T_{\mathrm{re}}$ and heart rate decreased mainly in the middle of the training while in the passive HA condition those values decreased at the end. Adaptive changes appearing later in the protocol for $\mathrm{HA}_{\mathrm{SUIT}}$ are indeed not surprising considering that the increase in $T_{\text {re }}$ for HAsuIT during the first $1 \mathrm{~h}$ was only $0.5 \pm 0.2{ }^{\circ} \mathrm{C}$, but it was accelerated during the second $1 \mathrm{~h}$ reaching $1.0 \pm 0.1{ }^{\circ} \mathrm{C}$. When utilizing only a skin-heating suit, "sufficiently overloading thermal impulses" that exceed an adaptation threshold, which is known to be the requirement for HA [30] seems to be achieved by $2 \mathrm{~h}$ but not by $1 \mathrm{~h}$. In addition, during the 2-h protocol AUC of $T_{\text {re }}$ for $\mathrm{HA}_{\mathrm{EXE}+\mathrm{SUIT}}$ was significantly greater than that for $\mathrm{HA}_{\mathrm{SUIT}}$, which may account for the earlier adaptation growth found during the protocol in $\mathrm{HA}_{\mathrm{EXE}+\mathrm{SUIT}}$.

Comparisons of the timing of HA induction in terms of reduction in $T_{\text {re }}$ and heart rate during the protocol for $\mathrm{HA}_{\mathrm{EXE}+\mathrm{SUIT}}$ with those found in $\mathrm{HA}_{\mathrm{EXE}}$ and HA $\mathrm{H}_{\text {SUIT }}$ make it more reasonable to interpret that the changes are due to the combined effects of exercise and passive heating: it appeared from the end of exercise to the early stage of passive heating. During exercise, $\mathrm{HA}_{\mathrm{EXE}}$ also elicited lower $T_{\text {re }}$ and heart rate, as well known [31, 32], but it did not last $\left(T_{\text {re }}\right)$ or only intermittently appeared after exercise stopped (heart rate). The decreases of $T_{\text {re }}$ and heart rate during the early phase of suit donning in $\mathrm{HA}_{\text {EXE+SUIT }}$ are less likely to be solely caused by the skin-heating, because in $\mathrm{HA}_{\text {SUIT }}$ such reduction in $T_{\text {re }}$ and heart rate appeared at the end of the heat acclimation protocol. Therefore, it is more likely that as previously suggested $[15,23]$, the post-exercise passive heating further induced actively induced HA responses by maintaining greater thermal impulses after exercise using the suit. This synergic effect of exercise and suit donning seems to have facilitated improvement in heat loss mechanism leading to decreased heat storage [33] as well as in reduction of cardiovascular strain, whose mechanisms are known to be associated with (1) plasma volume expansion, (2) blood volume redistribution, (3) enhanced skin cooling, (4) increase in venous tone, and (5) less stimulated sympathetic nervous activity [17, 34].

Another point to bear in mind is that $\mathrm{HA}_{\text {SUIT }}$ showed adaptations earlier than $\mathrm{HA}_{\mathrm{EXE}+\mathrm{SUIT}}$ did except for the sweat responses. On day $5, \mathrm{HA}_{\text {SUIT }}$ already showed reduction in $T_{\text {re }}$ and heart rate contributing to reduction in PSI, in accordance with the findings of previous studies suggesting that less than 7 days of training are enough for body core temperature reduction [10, 35]; decrease in heart rate appears within the first 4-5 days $[10,17]$. Lowest PSI on day 5 not on day 10 is likely to result from lower heart rate on day 5 than day 10, because $T_{\text {re }}$ on day 5 and day 10 were not different. 
Seemingly diminishing effects of wearing the suit on inducing heat adaptive changes in heart rate may be attributable to the constant work-rate model of this HA strategy. Albeit practical, fixed endogenous, and exogenous thermal impulses have been pointed out to provide stimuli not strong enough for further adaptations, as HA gradually developed [30]. It will be worthy to be further investigated to examine whether reduction in heart rate obtained by wearing the suit adopting the controlled hyperthermia model, which is considered optimal in terms of addressing the aforementioned point, will not be attenuated.

The later expression of elevated whole-body sweat rate for $\mathrm{HA}_{\text {SUIT }}$ might be explicable by the warm humid microclimate formed inside the suit. When sweat adaptations occur at a peripheral level, it is seldom related to the number of activated eccrine glands but due to higher efficiency and larger sizes of sweat glands [36]. In a hot and humid environment, sweating adaptations are largely associated with hidromeiosis, which decreases the mean output of each sweat gland of wetted skin. Fox et al. [37] demonstrated that higher whole-body sweat rate induced in humid heat acclimation is linked with reduction in hidromeiosis. Candas et al. [38] also suggested that initial oversweating as well as delay in the occurrence of sweat reduction takes place in acclimated individuals. Therefore, it is probable that in the more poorly ventilated condition ( $\mathrm{HA}_{\mathrm{SUIT}}$ ) due to the longer duration of suit donning, the eccrine glands had to develop not only morphological and functional changes but also greater resistance to hidromeiosis, in order to show more profuse sweating. The different tendencies in $T_{\text {re }}$ or heart rate (earlier in $\mathrm{HA}_{\text {SUIT }}$ ) and sweat response (later in $\mathrm{HA}_{\text {SUIT }}$ ) may be accounted for by the consensus that hidromeiosis and its adaptive changes are peripheral phenomenon and not related to central factors [38, 39]. Finally, 10 days is not a surprisingly long period for achieving an elevation in sweat rate as longer term HA interventions (at least 14 days were recommend by Tyler et al. [10]) have been shown to be appropriate for achieving sweat adaptations.

\section{Conclusions}

We explored the effects of passive heating using a waterperfused suit on heat adaptive changes for 10 days. The novel finding of the present study is that the postexercise and passive strategies that heated the skin using the suit (water inflow temperature $44.2-44.3{ }^{\circ} \mathrm{C}$ ) was more effective than the mild exercise (1-h walking at 6 $\mathrm{km} \mathrm{h}^{-1}$ ), in inducing adaptive changes in sweat and perceptual responses. In this regard, we can recommend to those who are not able to exercise in hot environments, utilize a water-perfused suit for at least $2 \mathrm{~h}$ daily for 10 days to induce heat acclimation. The elderly or the disabled may be the population who can benefit from using this newly proposed heat acclimation protocol. However, it should be carefully applied to the old or further investigation for them is needed because the present findings were found for the younger subjects. Elite athletes who want intensified heat acclimation could also incorporate this novel method into their training programs. When choosing between passive and post-exercise strategies, optimal heat acclimation responses for the specific acclimation period should be considered.

\section{Abbreviations}

HA: Heat acclimation; HAEXE: Heat acclimation with exercise; HAEXE+SUIT: Heat acclimation with exercise and post-exercise passive heating using a waterperfused suit; $\mathrm{HA}_{\text {surt }}$ : Heat acclimation with passive heating using a waterperfused suit; HR: Heart rate; HF: Heat flow; PSI: Physiological strain index; RPE: Rating of perceived exertion; $T_{\text {core }}$ : Body core temperature; $T_{\text {re: }}$ Rectal temperature; $T_{\text {wi }}$ : Water inflow temperature; $T_{\text {wo }}$ : Water outflow temperature; $\mathrm{VO}_{2 \text { max }}$ : Maximal oxygen uptake

\section{Acknowledgements}

We give our thanks to the subjects for participating in the present study. We are also grateful to Andrew Gorski and Yoon-Jeong Hur for their technical support.

\section{Authors' contributions}

The contributions of each author are as follows: $Y$ developed the idea of this study, planned the method, conducted the experiments, interpreted the results, analyzed the data, and prepared the manuscript. SH and J performed the experiments with $Y$ and JY. JY directed the idea development and experiments, interpreted the results with $Y$, and contributed to writing the final manuscript. All authors read and approved the final manuscript.

\section{Funding}

This work was supported by the National Research Foundation of Korea (NRF) grant funded by the Korea government (MSIT) (No.

2019R1A2C2006961), funded by the Korea Meteorological Administration Research and Development Program, "Advanced Research on Biometeorology and Industrial Meteorology" (No. 1365003004).

\section{Availability of data and materials}

The datasets used and/or analyzed during the current study are available from the corresponding author on reasonable request.

\section{Ethics approval and consent to participate}

All procedure involving human participants were performed in accordance with the ethical standards of the institutional research committee. The present study was approved by the Institutional Review Board of Seoul National University (IRB No. 1905/003-008; Jan 22, 2019). Informed consent was obtained from all participants in the present study, prior to experiments. All trials began after the registration date.

\section{Consent for publication}

Not applicable

\section{Competing interests}

The authors declare that they have no competing interests.

\section{Author details}

${ }^{1}$ Department of Textiles, Merchandising and Fashion Design, College of Human Ecology, Seoul National University, COMFORT Laboratory, Bld. \# 222-Rm. \# 306, 1 Gwanak-ro, Gwanak-gu, Seoul 08826, Korea. ²Department of Physical Education, Seoul National University, Seoul, Korea. ${ }^{3}$ Research Institute of Human Ecology, Seoul National University, Seoul, Korea. 
Received: 10 January 2020 Accepted: 11 March 2020

Published online: 14 April 2020

\section{References}

1. Hess KL, Wilson TE, Sauder CL, Gao Z, Ray CA, Monahan KD. Aging affects the cardiovascular responses to cold stress in humans. J Appl Physiol. 2009; 107(4):1076-82.

2. Nielsen B, Savard G, Richter E, Hargreaves M, Saltin B. Muscle blood flow and muscle metabolism during exercise and heat stress. J Appl Physiol. 1990;69(3):1040-6

3. Wilson TE, Tollund C, Yoshiga CC, Dawson EA, Nissen P, Secher NH, Crandall CG. Effects of heat and cold stress on central vascular pressure relationships during orthostasis in humans. J Physiol. 2007:585(1):279-85.

4. Wilson TE, Gao Z, Hess KL, Monahan KD. Effect of aging on cardiac function during cold stress in humans. Am J Physiol Regul Integr Comp Physiol. 2010;298(6):R1627-33.

5. Filingeri $D$, Morris NB, Jay O. Warm hands, cold heart: progressive wholebody cooling increases warm thermosensitivity of human hands and feet in a dose-dependent fashion. Exp Physiol. 2017;102(1):100-12.

6. Yamazaki F, Sone R. Different vascular responses in glabrous and nonglabrous skin with increasing core temperature during exercise. Eur J Appl Physiol. 2006;97(5):582-90.

7. Rowell LB, Brengelmann GL, Murray JA, Kraning KK 2nd, Kusumi F. Human metabolic responses to hyperthermia during mild to maximal exercise. J Appl Physiol. 1969;26(4):395-402.

8. Ostrow S, Van DE, Whitacre M, Aisner J, Simon R, Wiernik PH. Physiologic response and toxicity in patients undergoing whole-body hyperthermia for the treatment of cancer. Cancer Treat Rep. 1981;65(3-4):323-5.

9. Neff D, Kuhlenhoelter AM, Lin C, Wong BJ, Motaganahalli RL, Roseguini BT Thermotherapy reduces blood pressure and circulating endothelin-1 concentration and enhances leg blood flow in patients with symptomatic peripheral artery disease. Am J Physiol Regul Integr Comp Physiol. 2016; 311(2):R392-400

10. Tyler CJ, Reeve T, Hodges GJ, Cheung SS. The effects of heat adaptation on physiology, perception and exercise performance in the heat: a metaanalysis. Sports Med. 2016;46(11):1699-724.

11. Shin YO, Lee JB, Min YK, Yang HM. Heat acclimation affects circulating levels of prostaglandin E2, COX-2 and orexin in humans. Neurosci Lett. 2013;542: 17-20.

12. Pallubinsky $H$, Schellen $L$, Kingma B, Dautzenberg B, van Baak MA, van Marken Lichtenbelt WD. Thermophysiological adaptations to passive mild heat acclimation. Temperature. 2017;4(2):176-86

13. Stanley J, Halliday A, D'Auria S, Buchheit M, Leicht AS. Effect of sauna-based heat acclimation on plasma volume and heart rate variability. Eur J Appl Physiol. 2015;115(4):785-94.

14. Heathcote SL, Hassmén P, Zhou S, Stevens CJ. Passive heating: reviewing practical heat acclimation strategies for endurance athletes. Front Physiol. 2018:9:1-12

15. Daanen HA, Racinais S, Périard JD. Heat acclimation decay and re-induction: a systematic review and meta-analysis. Sports Med. 2018;48(2):409-30.

16. Kenny GP, Jay O. Thermometry, calorimetry, and mean body temperature during heat stress. Compr Physiol. 2011;3(4):1689-719.

17. Périard JD, Racinais S, Sawka MN. Adaptations and mechanisms of human heat acclimation: applications for competitive athletes and sports. Scand J Med Sci Sports. 2015;25:20-38.

18. Lee JY, Choi JW, Kim H. Determination of body surface area and formulas to estimate body surface area using the alginate method. J Physiol Anthropol. 2008:27(2):71-82

19. Borg GA. Psychophysical bases of perceived exertion. Med sci sports exerc 1982;14(5):377-81.

20. Moran DS, Shitzer A, Pandolf KB. A physiological strain index to evaluate heat stress. Am J Physiol Regul Integr Comp Physiol. 1998:275(1):R129-34.

21. Stachenfeld NS. Acute effects of sodium ingestion on thirst and cardiovascular function. Curr Sport Med Rep. 2008;7(4):S7.

22. Convertino VA. Heart rate and sweat rate responses associated with exercise-induced hypervolemia. Med Sci Sports Exerc. 1983;15(1):77-82.

23. Zurawlew MJ, Walsh NP, Fortes MB, Potter C. Post-exercise hot water immersion induces heat acclimation and improves endurance exercise performance in the heat. Scand J Med Sci Sports. 2016;26(7):745-54.
24. Scoon GS, Hopkins WG, Mayhew S, Cotter JD. Effect of post-exercise sauna bathing on the endurance performance of competitive male runners. J Sci Med Sport. 2007;10(4):259-62.

25. Armada-da-Silva PA, Woods J, Jones DA. The effect of passive heating and face cooling on perceived exertion during exercise in the heat. Eur J Appl Physiol. 2004;91(5-6):563-71.

26. Cheuvront SN, Kenefick RW, Montain SJ, Sawka MN. Mechanisms of aerobic performance impairment with heat stress and dehydration. J Appl Physiol. 2010:109(6):1989-95.

27. Stevens CJ, Mauger AR, Hassmen P, Taylor L. Endurance performance is influenced by perceptions of pain and temperature: theory, applications and safety considerations. Sports Med. 2018;48(3):525-37.

28. Nielson B, Hales JR, Strange S, Christensen NJ, Warberg J, Saltin B. Human circulatory and thermoregulatory adaptations with heat acclimation and exercise in a hot, dry enviornment. J Appl Physiol. 1993:460(1):467-85.

29. Gonzalez RR. Models useful for predicting human responses to the environment: application to hazard material operations. J Hum Environ Syst. 2000;4(1):1-10.

30. Taylor NA. Human heat adaptation. Compr Physiol. 2011;4(1):325-65.

31. Périard JD, Travers GJ, Racinais S, Sawka MN. Cardiovascular adaptations supporting human exercise-heat acclimation. Auton Neurosci. 2016;196: $52-62$.

32. Casadio JR, Kilding AE, Cotter JD, Laursen PB. From lab to real world: heat acclimation considerations for elite athletes. Sport med. 2017:47(8):1467-76.

33. Buono MJ, Heaney JH, Canine KM. Acclimation to humid heat lowers resting core temperature. Am J Physiol Regul Integr Comp Physiol. 1998; 274(5):R1295-9.

34. Hodge D, Jones D, Martinez R, Buono MJ. Time course of the attenuation of sympathetic nervous activity during active heat acclimation. Auton Neurosci. 2013;177(2):101-3.

35. Robinson S, Turrell ES, Belding HS, Horvath SM. Rapid acclimatization to work in hot climates. Am J Physiol. 1943;140(2):168-76.

36. Hori S. Adaptation to heat. Jpn J Physiol. 1995:45(6):921-46.

37. Fox RH, Goldsmith R, Hampton IFG, Lewis HE. The nature of the increase in sweating capacity produced by heat acclimatization. J Physiol. 1964;171(3): $368-76$

38. Candas V, Libert JP, Vogt JJ. Effect of hidromeiosis on sweat drippage during acclimation to humid heat. Am J Physiol Regul Integr Comp Physiol. 1980;44(2):123-33.

39. Ogawa T, Asayama M, Miyagawa T. Effects of sweat gland training by repeated local heating. Jpn J Physiol. 1982;32(6):971-81.

\section{Publisher's Note}

Springer Nature remains neutral with regard to jurisdictional claims in published maps and institutional affiliations.

Ready to submit your research? Choose BMC and benefit from

- fast, convenient online submission

- thorough peer review by experienced researchers in your field

- rapid publication on acceptance

- support for research data, including large and complex data types

- gold Open Access which fosters wider collaboration and increased citations

- maximum visibility for your research: over $100 \mathrm{M}$ website views per year

At BMC, research is always in progress.

Learn more biomedcentral.com/submission 